PROCEEDINGS OF THE

AMERICAN MATHEMATICAL SOCIETY

Volume 137, Number 6, June 2009, Pages 1849-1853

S 0002-9939(09)09786-X

Article electronically published on January 9, 2009

\title{
MINIMAL POLYNOMIAL OF AN EXPONENTIAL AUTOMORPHISM OF $\mathbb{C}^{n}$
}

\author{
JAKUB ZYGADEO
}

(Communicated by Bernd Ulrich)

\begin{abstract}
We show that the minimal polynomial of a polynomial exponential automorphism $F$ of $\mathbb{C}^{n}$ (i.e. $F=\exp (D)$ where $D$ is a locally nilpotent derivation) is of the form $\mu_{F}(T)=(T-1)^{d}$, with $d=\min \left\{m \in \mathbb{N}: D^{\circ m}\left(X_{i}\right)=\right.$ 0 for $i=1, \ldots, n\}$.
\end{abstract}

\section{INTRODUCTION}

Let $k$ be a field of characteristic zero and let $A$ be a $k$-algebra. Recall that a $k$ derivation of $A$ is a $k$-linear mapping $D: A \rightarrow A$ fulfilling the Leibniz rule $D(a b)=$ $D(a) b+a D(b)$. We will write $D^{\circ n}$ for the $n$-th iterate of $D$, i.e. $D^{\circ n}=D \circ D^{\circ(n-1)}$ and $D^{\circ 0}=I$ - the identity. If for every $a \in A$ there exists $n=n(a) \in \mathbb{N}$ such that $D^{\circ n}(a)=0$, the derivation $D$ is called locally nilpotent.

If $D$ is a locally nilpotent derivation of $A$, we define the exponential of $D, \exp (D)$, by the formula

$$
\exp (D)(a):=\sum_{i=0}^{\infty} \frac{1}{i !} D^{\circ i}(a)
$$

It is easy to see that $\exp (D): A \rightarrow A$ is a $k$-endomorphism of $A$. One can also check that if locally nilpotent $k$-derivations $D$ and $E$ commute (i.e. $D \circ E=E \circ D$ ), then $\exp (D) \circ \exp (E)=\exp (E) \circ \exp (D)=\exp (D+E)$. Therefore, $\exp (D)$ is an automorphism of $A$ with inverse $\exp (D)^{-1}=\exp (-D)$. In this paper we prove the following:

Theorem. Let $D$ be a locally nilpotent derivation of $\mathbb{C}\left[X_{1}, \ldots, X_{n}\right]$, and define $F:=\left(\exp (D)\left(X_{1}\right), \ldots, \exp (D)\left(X_{n}\right)\right): \mathbb{C}^{n} \rightarrow \mathbb{C}^{n}$ and $d:=\min \left\{m \in \mathbb{N}: D^{\circ m}\left(X_{i}\right)=\right.$ 0 for $i=1, \ldots, n\}$. Then the minimal polynomial for $F$ equals $\mu_{F}(T)=(T-1)^{d}=$ $\sum_{j=0}^{d}(-1)^{d-j}\left(\begin{array}{l}d \\ j\end{array}\right) T^{j}$ (i.e. the mapping $\mu_{F}(F)=\sum_{j=0}^{d}(-1)^{d-j}\left(\begin{array}{c}d \\ j\end{array}\right) F^{\circ j}$ is zero and $p(F) \neq 0$ for any polynomial $p \in \mathbb{C}[T] \backslash\{0\}$ of degree less than $d)$. In particular, we have the following formula for the inverse of $F$ :

$$
F^{-1}=\sum_{j=0}^{d-1}(-1)^{j}\left(\begin{array}{c}
d \\
j+1
\end{array}\right) F^{\circ j} .
$$

Received by the editors January 7, 2008

2000 Mathematics Subject Classification. Primary 14R10; Secondary 13N15.

Key words and phrases. Polynomial automorphism, locally nilpotent derivation, minimal polynomial.

(C)2009 American Mathematical Society Reverts to public domain 28 years from publication 


\section{Preparatory steps}

First, we will prove two simple lemmas:

Lemma 1. Let $A$ be a $k$-algebra, $D$ a locally nilpotent $k$-derivation of $A$ and $a \in A$. If for some $m \geq 1$ and $\alpha_{0}, \ldots, \alpha_{m-1} \in k$ there is an equality

$$
D^{\circ m}(a)=\sum_{i=0}^{m-1} \alpha_{i} D^{\circ i}(a),
$$

then $D^{\circ m}(a)=0$.

Proof. We will proceed by induction on $m$. When $m=1$ we have $D(a)=\alpha_{0} a$ and the result is well known (even for $\alpha_{0} \in A$, if $A$ has no zero divisors - see for example [2], Prop. 1.3.32), but we will prove it for the sake of completeness. If $D(a)=\alpha_{0} a$, then $D^{\circ n}(a)=D^{\circ(n-1)}\left(\alpha_{0} a\right)=\ldots=D\left(\alpha_{0}^{n-1} a\right)=\alpha_{0}^{n} a$ for all $n \in \mathbb{N}$. Because $D$ is locally nilpotent, we must have $D^{\circ n}(a)=0$ for some $n$ and consequently $\alpha_{0}=0$ or $a=0$. Now let $m>1$ and assume that the lemma holds for all $m^{\prime}<m$. Suppose $D^{\circ m}(a) \neq 0$ and let $M \in \mathbb{N}$ be such that $D^{\circ M}(a)=0$ and $D^{\circ(M-1)}(a) \neq 0$ (note $M>m$ ). Set $i_{0}:=\max \left\{0 \leq i<m: \alpha_{i} \neq 0\right\}$; then we can write $0=D^{\circ M}(a)=D^{\circ(M-m)}\left(D^{\circ m}(a)\right)=D^{\circ(M-m)}\left(\sum_{i=0}^{i_{0}} \alpha_{i} D^{\circ i}(a)\right)=$ $\sum_{i=0}^{i_{0}} \alpha_{i} D^{\circ i}\left(D^{\circ(M-m)}(a)\right)$. Let $a^{\prime}:=D^{\circ(M-m)}(a)$. Because $\alpha_{i_{0}} \neq 0$, we have $D^{\circ i_{0}}\left(a^{\prime}\right)=-\sum_{i=0}^{i_{0}-1} \frac{\alpha_{i}}{\alpha_{i_{0}}} D^{\circ i}\left(a^{\prime}\right)$ and since $i_{0}<m$, we obtain $D^{\circ i_{0}}\left(a^{\prime}\right)=0$ by the induction hypothesis - this is a contradiction with $D^{\circ i_{0}}\left(a^{\prime}\right)=D^{\circ\left(M-m+i_{0}\right)}(a) \neq$ 0 .

Lemma 2. Let $d>0, i \in \mathbb{N}$ and define

$$
\beta_{d, i}:=\sum_{m=0}^{d}(-1)^{m}\left(\begin{array}{c}
d \\
m
\end{array}\right) m^{i} .
$$

We have $\beta_{d, i}=0$ if and only if $i<d$.

Proof. Equality $\beta_{d, 0}=0$ follows from expansion of $(1-1)^{d}=0$, and the case $d=1$ is obvious. Let $d>1, i>0$ and proceed by induction on $d$. We have

$$
\begin{aligned}
\beta_{d, i} & =\sum_{m=1}^{d}(-1)^{m} d\left(\begin{array}{c}
d-1 \\
m-1
\end{array}\right) m^{i-1}=-d \sum_{m=0}^{d-1}(-1)^{m}\left(\begin{array}{c}
d-1 \\
m
\end{array}\right)(m+1)^{i-1} \\
& =-d \sum_{j=0}^{i-1}\left(\begin{array}{c}
i-1 \\
j
\end{array}\right)\left(\sum_{m=0}^{d-1}(-1)^{m}\left(\begin{array}{c}
d-1 \\
m
\end{array}\right) m^{j}\right)=-d \sum_{j=0}^{i-1}\left(\begin{array}{c}
i-1 \\
j
\end{array}\right) \beta_{d-1, j}
\end{aligned}
$$

and for $i<d$ we can conclude by using the induction hypothesis, because all $\beta_{d-1, j}=0$. To deal with the case $i \geq d$, note that $\beta_{1, i}=-1$ for $i \geq 1$, so $\beta_{2, i}=-2 \sum_{j=0}^{i-1}\left(\begin{array}{c}i-1 \\ j\end{array}\right) \beta_{1, j}>0$ for $i \geq 2$. Proceeding in this way, we see that $(-1)^{d} \beta_{d, i}>0$ for $i \geq d$.

From now on we will focus our attention on the case where $k=\mathbb{C}$ (though the result is valid for any field of characteristic zero) and $A=\mathbb{C}\left[X_{1}, \ldots, X_{n}\right]$ - the ring of polynomials in $n$ variables. It can be shown that every $\mathbb{C}$-derivation $D$ of $A$ is of the form $D=\sum_{i=1}^{n} f_{i} \partial_{x_{i}}$ for some $f_{1}, \ldots, f_{n} \in A$, where $\partial_{x_{i}}=\frac{\partial}{\partial X_{i}}$ is the standard differential with respect to $X_{i}$. 
If $\Phi: A \rightarrow A$ is a $\mathbb{C}$-endomorphism of $A$, one can define a polynomial mapping $\Phi_{*}: \mathbb{C}^{n} \rightarrow \mathbb{C}^{n}$ by

$$
\Phi_{*}=\left(\Phi\left(X_{1}\right), \ldots, \Phi\left(X_{n}\right)\right) .
$$

Obviously $I_{*}=I$ and $(\Phi \circ \Psi)_{*}=\Psi_{*} \circ \Phi_{*}$, so each $\mathbb{C}$-automorphism $\Phi$ of $A$ gives rise to a polynomial automorphism $\Phi_{*}$ of the affine space $\mathbb{C}^{n}$. In particular, if $D$ is a locally nilpotent derivation of $A$ and $\Phi=\exp (D)$, we have an automorphism $F=\exp (D)_{*}=\left(\exp (D)\left(X_{1}\right), \ldots, \exp (D)\left(X_{n}\right)\right)$ of $\mathbb{C}^{n}$, called the exponential automorphism.

In [1, the following class of polynomial automorphisms is considered: Let $F=$ $\left(F_{1}, \ldots, F_{n}\right)$ be a polynomial automorphism of $\mathbb{C}^{n}$. If there is an univariate polynomial $p(T) \in \mathbb{C}[T] \backslash\{0\}$ such that $p(F)=0$ (i.e. for $p(T)=a_{0}+a_{1} T+\ldots+a_{m} T^{m}$ this means $\left.a_{0} I+a_{1} F+\ldots+a_{m} F^{\circ m}=0\right)$; then $F$ is called locally finite.

It is easy to see that the set $I_{F}:=\{p \in \mathbb{C}[T]: p(F)=0\}$ forms an ideal in $\mathbb{C}[T]$; its monic generator will be called the minimal polynomial for $F$ and denoted by $\mu_{F}$. The paper [1] gives many equivalent conditions for $F$ to be locally finite and presents a formula for a polynomial $p(T)$ such that $p(F)=0$ provided $F(0)=0$ (see [1, Th. 1.2). Unfortunately, there is no such result when $F(0) \neq 0$ and it is not easy to find the minimal polynomial $\mu_{F}$ either. We solve this problem for exponential automorphisms of $\mathbb{C}^{n}$ in the following section.

\section{The Main Result And its CONSEQUences}

Theorem (Main theorem). Let $D$ be a locally nilpotent derivation of $\mathbb{C}\left[X_{1}, \ldots, X_{n}\right]$, and define $F:=\exp (D)_{*}$ and $d:=\min \left\{m \in \mathbb{N}: D^{\circ m}\left(X_{i}\right)=0\right.$ for $\left.i=1, \ldots, n\right\}$. Then the minimal polynomial for $F$ equals $\mu_{F}(T)=(T-1)^{d}$.

Proof. Note that for $m \in \mathbb{N}$, we have $F^{\circ m}=\left(\exp (D)^{\circ m}\right)_{*}=\exp (m D)_{*}$ (because $D$ commutes with $D)$; so if $F=\left(F_{1}, \ldots, F_{n}\right)$, then

$$
\left(F^{\circ m}\right)_{j}=\sum_{i=0}^{d-1} \frac{1}{i !}(m D)^{\circ i}\left(X_{j}\right)=\sum_{i=0}^{d-1} \frac{1}{i !} m^{i} D^{\circ i}\left(X_{j}\right), \quad j=1, \ldots, n .
$$

Since

$$
\begin{aligned}
\sum_{m=0}^{d}(-1)^{m}\left(\begin{array}{c}
d \\
m
\end{array}\right)\left(F^{\circ m}\right)_{j} & =\sum_{i=0}^{d-1} \frac{1}{i !}\left(\sum_{m=0}^{d}(-1)^{m}\left(\begin{array}{c}
d \\
m
\end{array}\right) m^{i}\right) D^{\circ i}\left(X_{j}\right) \\
& =\sum_{i=0}^{d-1} \frac{1}{i !} \beta_{d, i} D^{\circ i}\left(X_{j}\right),
\end{aligned}
$$

we conclude by Lemma 2 that $\sum_{m=0}^{d}(-1)^{m}\left(\begin{array}{c}d \\ m\end{array}\right) F^{\circ m}=0$. This argument shows that the polynomial $(1-T)^{d} \in I_{F}=\{p \in \mathbb{C}[T]: p(F)=0\}$. To prove minimality of its degree, assume for example that $d=\min \left\{m \in \mathbb{N}: D^{\circ m}\left(X_{1}\right)=0\right\}$ and suppose that $\mu_{F}(T)=(T-1)^{e}$ for some $e<d$. Then $0=(-1)^{e}\left(\mu_{F}(F)\right)_{1}=$ $\sum_{m=0}^{e}(-1)^{m}\left(\begin{array}{c}e \\ m\end{array}\right)\left(F^{\circ m}\right)_{1}=\sum_{i=0}^{d-1} \frac{1}{i !} \beta_{e, i} D^{\circ i}\left(X_{1}\right)$ and $\beta_{e, d-1} \neq 0$ by Lemma2 Therefore $D^{\circ(d-1)}\left(X_{1}\right)=-\sum_{i=0}^{d-2} \frac{1}{i !} \beta_{e, i} \beta_{e, d-1} D^{\circ i}\left(X_{1}\right)$, and, owing to Lemma 1, we obtain $D^{\circ(d-1)}\left(X_{1}\right)=0$ despite the definition of $d$, a contradiction. 
Corollary. Since $\mu_{F}(F)=0$, we have $I=\left(\sum_{m=1}^{d}(-1)^{m-1}\left(\begin{array}{c}d \\ m\end{array}\right) F^{\circ(m-1)}\right) \circ F$ and therefore the inverse of $F$ is given by

$$
F^{-1}=\sum_{m=0}^{d-1}(-1)^{m}\left(\begin{array}{c}
d \\
m+1
\end{array}\right) F^{\circ m} .
$$

Remark 1. The famous Nagata automorphism of $\mathbb{C}^{3}$ (see [3]) defined by $N=$ $\left(X-2 Y \sigma-Z \sigma^{2}, Y+Z \sigma, Z\right)$ where $\sigma=X Z+Y^{2}$ can be seen as an exponential of a locally nilpotent derivation $D=-2 Y \sigma \partial_{x}+Z \sigma \partial_{y}$ of $\mathbb{C}[X, Y, Z]$. It is easy to check that $D(\sigma)=0$ and $D^{\circ 3}(X)=D^{\circ 3}(Y)=D^{\circ 3}(Z)=0$, so the main theorem gives $\mu_{N}(T)=(T-1)^{3}$, whereas by [1], Th. 1.2 we only get that $p(T)=(T-1)^{55} \in I_{N}$.

Remark 2. Let $d \geq 2$ and $D=Y^{d-2} \partial_{x}+\partial_{y}$. Obviously $D$ is a locally nilpotent derivation of $\mathbb{C}[X, Y]$ and $D^{\circ d}(X)=D^{\circ d}(Y)=0$ ( $d$ is minimal). If we let $F=$ $\exp (D)_{*}$, then $\mu_{F}(T)=(T-1)^{d}$ by the main theorem. Since clearly $\operatorname{deg} F=d-2$, this shows that the estimate $\operatorname{deg} \mu_{F} \leq \operatorname{deg} F+1$ ([1, Th. 4.2) need not hold if $F(0) \neq 0$.

Remark 3. Recall that if $P=\left(P_{1}, \ldots, P_{n}\right): \mathbb{C}^{n} \rightarrow \mathbb{C}^{n}$ is a polynomial mapping, then $P^{*}$ given by $P^{*}\left(X_{i}\right):=P_{i} \in \mathbb{C}\left[X_{1}, \ldots, X_{n}\right]$ defines a $\mathbb{C}$-endomorphism of $\mathbb{C}\left[X_{1}, \ldots, X_{n}\right]$. Let $F=(X+g(Y, Z), Y+h(Z), Z)$ be an upper triangular automorphism of $\mathbb{C}^{3}(g \in \mathbb{C}[Y, Z], h \in \mathbb{C}[Z])$. If $g=0$ or $h=0$, then $F$ is easily seen to be an exponential of a locally nilpotent derivation of $\mathbb{C}[X, Y, Z]$. So let us suppose that $g \neq 0$ and $h \neq 0$. We will show that the minimal polynomial for $F$ equals $\mu_{F}(T)=(T-1)^{d}$, where $d:=2+\operatorname{deg}_{Y} g$, and that therefore in this case we also have $F=\exp (D)_{*}$ (see [1, Th. 2.3) for the locally nilpotent derivation $D$ given by the formula $D=\sum_{m=1}^{+\infty} \frac{(-1)^{m+1}}{m}\left(F^{*}-I^{*}\right)^{\circ m}$ (cf. 2], Ch. 2). Obviously $D(Z)=0$, $D(Y)=h(Z)$ and one can use the above formula to evaluate $D(X)$. Note that if we can show that the minimal polynomial has degree $d$, then only the first $d-1$ summands are nonzero. Write $g(Y, Z)=\sum_{i=0}^{d-2} Y^{i} g_{i}(Z)$. Iterating $F$, we get

$$
\begin{aligned}
F^{\circ m} & =\left(X+\sum_{j=0}^{m-1} \sum_{i=0}^{d-2}(Y+j h(Z))^{i} g_{i}(Z), Y+m h(Z), Z\right) \\
& =\left(X+\sum_{i=0}^{d-2} g_{i}(Z) \sum_{k=0}^{i}\left(\begin{array}{l}
i \\
k
\end{array}\right) Y^{i-k} h(Z)^{k} \sum_{j=0}^{m-1} j^{k}, Y+m h(Z), Z\right) .
\end{aligned}
$$

Let $s_{k}(m):=\sum_{j=0}^{m-1} j^{k}$ and note that $s_{k}$ is a polynomial in $m$ of degree $k+1 \leq$ $d-1<d$. Therefore, Lemma 2 gives $\sum_{m=0}^{d}(-1)^{m}\left(\begin{array}{c}d \\ m\end{array}\right) s_{k}(m)=0$ for all $k \leq d-2$ and we can argue as in the proof of the main theorem (since $g_{d-2}(Z) h(Z)^{d-2} s_{d-2}(m)$ is the only term involving $m^{d-1}$, we must have $D^{\circ(d-1)}(X) \neq 0$ ). Consequently $\mu_{F}(T)=(T-1)^{d}$ and $F=\exp (D)_{*}$, where

$$
D=\left(\sum_{m=1}^{d-1} \sum_{i=1}^{m} \frac{(-1)^{i+1}}{m}\left(\begin{array}{c}
m \\
i
\end{array}\right)\left(\left(F^{\circ i}\right)_{1}-X\right)\right) \partial_{x}+h(Z) \partial_{y}
$$

and $d=2+\operatorname{deg}_{Y} g$. Note that $d$ is minimal and easily found in this case (there are obstacles to calculations of the minimal degree; cf. [1, Th. 1.2). 


\section{REFERENCES}

[1] J.-P. Furter, S. Maubach, Locally finite polynomial endomorphisms, J. Pure and Applied Algebra, 211(2) (2007), pp. 445-458. MR2340462 (2008e:14084)

[2] A. van den Essen, Polynomial Automorphisms and the Jacobian Conjecture, Progress in Math., vol. 190, Birkhäuser-Verlag, Basel, Boston, Berlin, 2000. MR1790619 (2001j:14082)

[3] M. Nagata, On the automorphism group of $k[x, y]$, in: Kyoto Univ. Lectures in Math., vol. 5, Kyoto University, Kinokuniya, Tokyo, 1972. MR0337962 (49:2731)

Institute of Mathematics, Jagiellonian University, Reymonta 4, 30-059 Kraków, POLAND

E-mail address: jakub.zygadlo@im.uj.edu.pl 